Artículo original

\title{
Genotipificación de aislamientos del complejo Mycobacterium tuberculosis mediante MIRU-VNTR, Cali, Colombia, 2013-2015
}

\author{
David Felipe García, Myriam Astudillo \\ Departamento de Microbiología, Universidad del Valle, Cali, Colombia
}

Introducción. La tuberculosis continúa siendo uno de los problemas de salud más importantes a nivel mundial y, con la infección por el virus de la inmunodeficiencia humana (HIV), constituye la principal causa de muerte por infecciones. En el 2016, se notificaron 6,3 millones de casos nuevos de la enfermedad.

Objetivo. Describir los patrones genéticos determinados mediante la genotipificación del número variable de repeticiones en tándem de unidades repetitivas interespaciadas de micobacterias (Mycobacterial Interspersed Repetitive Units - Variable Number of Tandem Repeats, MIRU-VNTR) en la población de estudio y compararlos con los hallados en otros estudios locales e internacionales.

Materiales y métodos. Mediante MIRU-VNTR, entre el 2013 y el 2015 se hizo la genotipificación de 105 muestras de ADN extraídas del esputo o de aislamientos en cultivo de $M$. tuberculosis provenientes de pacientes residentes en Cali con diagnóstico de tuberculosis pulmonar. La amplificación de 24 loci MIRU-VNTR se hizo por medio de la reacción en cadena de la polimerasa (PCR). Los amplicones resultantes se visualizaron por electroforesis en geles de agarosa (2\%) teñidos con SYBR Safe ${ }^{\mathrm{TM}}$. La asignación de los alelos se hizo con un análisis gráfico con el programa GelAnalyzer 2010. Los resultados obtenidos se analizaron con el algoritmo UPGMA y se compararon con las bases de datos internacionales MIRU-VNTRplus y SITVITWEB.

Resultados. Se genotipificaron por completo 62 de las muestras y se obtuvieron 58 perfiles diferentes de MIRU-VNTR. Al comparar con las bases de datos internacionales, su distribución por linajes fue la siguiente: 54,8 \% para el LAM, 25,8 \% para el Haarlem, 14,5\% para el S, $3,2 \%$ para el Beijing y $1,6 \%$ para el Cameroon. Los patrones MIRU-VNTR correspondieron a 20 tipos internacionales de MIRU (MIRU International Types, MIT) diferentes, y los más frecuentes fueron el MIT 190 y el MIT 110, con 22,6 y 6,5\%, respectivamente.

Conclusión. Estos resultados confirmaron hallazgos previos sobre el predominio de los linajes LAM y Haarlem en la ciudad y la presencia de los MIT encontrados en otra ciudad de Colombia.

Palabras clave: Mycobacterium tuberculosis; tuberculosis pulmonar; repeticiones de minisatélite.

Recibido: $08 / 08 / 17$

Aceptado: $27 / 08 / 18$

Publicado: $30 / 08 / 18$

Citación:

García D, Astudillo M. Genotipificación de aislamientos del complejo Mycobacterium tuberculosis mediante MIRU-VNTR, Cali, Colombia, 2013-2015. Biomédica. 2018;39(Supl.1):71-85 https://doi.org/10.7705/biomedica.v39i2.3924

\section{Correspondencia:}

Myriam Astudillo, Departamento de Microbiología, Universidad del Valle, Avenida $1 \mathrm{~N} \mathrm{~N}^{\circ}$ 3N-35, Cali, Colombia

Teléfono: (572) 6670327

myriam.astudillo@correounivalle.edu.co

Contribución de los autores:

David Felipe García: desarrollo de los

procedimientos experimentales, compilación de los resultados

Ambos autores participaron en el análisis y discusión de los resultados, y en la escritura del manuscrito.

Financiación:

El estudio fue financiado por el Departamento de Microbiología de la Universidad del Valle.

Conflicto de intereses:

Los autores declaran no tener conflictos de intereses.
MIRU-VNTR genotyping of Mycobacterium tuberculosis in a population of patients in Cali, Colombia, 2013-2015

Introduction: Tuberculosis continues to be one of the main public health problems in the world. Together with the HIV infection, it is one of the main causes of death due to infections worldwide. In 2016, 6.3 million new cases of the disease were reported.

Objective: To describe the genetic patterns determined by genotyping using variable-number tandem repeats of mycobacterial interspersed repetitive units (MIRU-VNTR) in the study population and compare them with other studies carried out in Cali, Colombia, and the world. Materials and methods: We genotyped a total of 105 DNA samples extracted from sputum or culture isolates of the Mycobacterium tuberculosis complex, which were obtained from pulmonary tuberculosis diagnosed patients over the period 2013-2015, in Cali. We performed PCR amplification of 24 loci by MIRU-VNTR on the DNA extracted from the samples. The amplicons were visualized in agarose gel electrophoresis $(2 \%)$ with SYBR Safe ${ }^{\mathrm{TM}}$ staining. Then, the alleles were designated by graphical analysis using the GelAnalyzer 2010 software. These results were analyzed using the UPGMA logarithm and compared with the registers from the MIRU-VNTR plus and SITVITWEB databases.

Results: We genotyped 62 of the samples completely and we obtained 58 different MIRUVNTR profiles. By comparing with the international databases, we determined the following distributions per lineage: LAM, 54.8\%; Haarlem,25.8\%; S, 14.5\%; Beijing, 3.2\%, and Cameroon, $1.6 \%$. The MIRU-VNTR patterns corresponded to 17 different MITs; the most frequent were MIT 190 and MIT 110, with $22.6 \%$ and $6.5 \%$, respectively.

Conclusions: These results demonstrated previous observations about the predominance of the LAM and Haarlem lineages in the city, and the presence of the MITs found in another city of Colombia.

Keywords: Mycobacterium tuberculosis; tuberculosis, pulmonary; minisatellite repeats. 
La tuberculosis continúa siendo uno de los problemas de salud más importantes en el mundo y, con la infección por el virus de inmunodeficiencia humana (HIV), constituye una de las principales causas de muerte por infecciones. En el 2016, se notificaron 6,3 millones de casos nuevos de la enfermedad (1).

Según la Organización Mundial de la Salud, la tuberculosis multirresistente se define como aquella resistente a los dos fármacos antituberculosos más potentes (isoniacida y rifampicina). Por otra parte, la tuberculosis 'ultrarresistente' lo es también a cualquier fluoroquinolona (como la levofloxacina o la moxifloxacina) y, por lo menos, a uno de los tres fármacos antituberculosos inyectables de segunda línea (amikacina, capreomicina o kanamicina) (2).

La creciente resistencia a los medicamentos antituberculosos en algunos países, y la relación existente entre las precarias condiciones de vida y de trabajo y la presencia del HIV en la población afectada, entre otros factores, han dificultado el control efectivo de esta enfermedad (3). La amenaza mundial de la tuberculosis ha servido de acicate para el despliegue de un esfuerzo global en la búsqueda de métodos eficaces para su vigilancia epidemiológica orientados al control y la disminución de su incidencia, el diagnóstico oportuno y el tratamiento adecuado de los casos ya notificados.

En el 2017, se notificaron al Sistema de Vigilancia en Salud Pública (Sivigila) de Colombia 14.480 casos de tuberculosis en todas sus formas, con una tasa de incidencia de 26,3 casos por 100.000 habitantes (4), pero con diferencias significativas entre los territorios, pues en algunos esta duplica el promedio nacional, lo cual refleja, a su vez, diferencias en las medidas de control y de vigilancia, así como en las dinámicas de transmisión.

El Valle del Cauca es uno de los departamentos con mayor número de casos notificados a nivel nacional. En el 2015, se notificaron cerca de 1.552 casos de tuberculosis pulmonar, con una incidencia de 29,8 casos por 100.000 habitantes (5). Cali se considera una ciudad con alto riesgo de tuberculosis dado que la incidencia es mayor a la observada en el territorio nacional. En el 2016, se encontró una incidencia de la forma pulmonar de 34 por 100.000 habitantes (6), con tendencia a aumentar, lo que constituye un complejo panorama para su control. A ello se suma el alto porcentaje de abandono del tratamiento $(15 \%)$ y los casos de infección concomitante de tuberculosis y HIV (16 a 18 \%) (5).

En este contexto, la tipificación molecular de los aislamientos del complejo Mycobacterium tuberculosis se acepta cada vez más como una herramienta útil que facilita el conocimiento de la epidemiología de la tuberculosis (7), y su prevención y control. La información que dicho análisis brinda ha contribuido a la comprensión de la dinámica de la transmisión y la patogenia de la enfermedad (8-10), y ha permitido establecer los probables factores de riesgo de la transmisión reciente y su alcance en una población, así como detectar la transmisión cuando no se ha sospechado tempranamente e identificar cepas resistentes a los medicamentos, confirmar la contaminación cruzada en el laboratorio, determinar los resultados falsos positivos en los cultivos y distinguir entre casos de reinfección o recaídas (11-13).

Se han desarrollado numerosos métodos para la genotipificación de cepas del complejo $M$. tuberculosis y para describir su estructura poblacional. La técnica desarrollada por van Embden (14), basada en el análisis de polimorfismos en la longitud de fragmentos de restricción (RFLP) y la detección de la variabilidad en la presencia y posición de la secuencia de inserción IS6110 
(RFLP-IS6110), era la técnica estándar para la tipificación de las micobacterias (15). Sin embargo, el tiempo y la laboriosidad que exigía impulsaron el desarrollo de métodos basados en la reacción en cadena de la polimerasa (PCR), aunque la técnica de van Embden sigue utilizándose en casos particulares (16).

La técnica de tipificación con oligonucleótidos espaciadores (spoligotyping) mediante PCR del locus DR (direct repeat) (17), así como el MIRU-VNTR (18), han reemplazado la prueba de RFLP-IS6110, ya que su complejidad técnica es menor, tienen portabilidad de resultados, y las bases de datos internacionales permiten la comparación con las colecciones de aislamientos de diversos orígenes geográficos, el intercambio de información, la visualización de la distribución global de los linajes genotípicos del complejo M. tuberculosis y el seguimiento de la dispersión de los aislamientos de alto riesgo por su virulencia y su resistencia a los medicamentos convencionales, así como información siempre creciente sobre múltiples marcadores genéticos $(19,20)$ y herramientas computacionales (21) de análisis. Ello genera una red para el estudio global de la epidemiología de la tuberculosis y permite un acercamiento a la exploración de los aspectos evolutivos del complejo M. tuberculosis (22).

En este estudio, se utilizó la técnica MIRU-VNTR para la genotipificación de 105 muestras de ADN obtenidas del esputo o de aislamientos clínicos de una población de pacientes con diagnóstico de tuberculosis pulmonar en Cali, con el objetivo de describir los patrones genéticos de $M$. tuberculosis en la población de estudio, compararlos con otros estudios llevados a cabo en esta ciudad y el mundo, y plantear hipótesis sobre las posibles asociaciones entre las agrupaciones encontradas en los aislamientos y las variables sociodemográficas.

\section{Materiales y métodos}

\section{Población de estudio}

Entre 2013 y 2015, se seleccionaron 105 pacientes atendidos en varias instituciones prestadoras de servicios de salud que participan en el Programa de Control de la Tuberculosis de la Secretaría de Salud Pública Municipal de Cali. En el marco de un convenio interadministrativo entre esa Secretaría y la Universidad del Valle, se obtuvieron las muestras de los pacientes, la ficha de la notificación epidemiológica del caso y el consentimiento informado.

Las muestras se procesaron en el Laboratorio de Micobacterias del Departamento de Microbiología de la Universidad del Valle. Se repitió la baciloscopia mediante tinción de Zielh-Nielsen en las muestras para confirmar la presencia de bacilos ácido-alcohol resistentes (BAAR) y, además, se las descontaminó con el método de Petroff modificado con hidróxido de sodio $(\mathrm{NaOH})$ y $\mathrm{N}$-acetilcisteína (23) para luego cultivarlas en medio LowënsteinJensen e incubarlas a $37^{\circ} \mathrm{C}$ hasta conseguir su crecimiento durante un máximo de 12 semanas, con el fin de ratificar el diagnóstico.

\section{Caracterización sociodemográfica y epidemiológica}

Se recolectaron los datos sociodemográficos y epidemiológicos de las fichas de notificación utilizadas para el ingreso de los pacientes al Programa de Control de la Tuberculosis de la Secretaría de Salud de la ciudad: edad, sexo, lugar de residencia, ocupación, condición de ingreso (caso nuevo, recaída o reingreso). Además, en el marco del convenio y con autorización del Programa, se tuvo acceso a los resultados de las pruebas de sensibilidad a los fármacos antituberculosos. 
Por otra parte, se tuvieron en cuenta condiciones de riesgo como el abandono del tratamiento, el contacto previo con personas con tuberculosis, y las condiciones médicas debilitantes del sistema inmunitario; entre estas últimas, se consideraron la desnutrición, el embarazo y la pertenencia a grupos con una alta tasa de transmisión, como habitantes de calle, farmacodependientes o infectados con HIV, entre otros.

Para garantizar la confidencialidad de los datos y para la protección de los pacientes, se utilizó un sistema de codificación numérica de identificación de todos los pacientes y sus muestras.

\section{Análisis moleculares}

Para la genotipificación se extrajo ADN a partir de las muestras de esputo de los pacientes. En los casos en que la concentración de ADN era menor de $10 \mathrm{ng} / \mu \mathrm{l}$, la extracción se hizo a partir de una unidad formadora de colonia (UFC) obtenida del cultivo.

Extracción de ADN. El ADN de las muestras (esputo o cultivo) se extrajo utilizando el estuche de extracción Genolyse ${ }^{\mathrm{TM}}$ (Hain Lifescience $\mathrm{GmbH}$, Nehren, Alemania). Se hicieron ensayos preliminares para comprobar la compatibilidad del ADN obtenido con la genotipificación (no se incluyen los datos). La calidad y la cantidad del ADN obtenido se evaluaron con el equipo NanoDrop ${ }^{\mathrm{TM}}$ (Thermo Scientific, Estados Unidos).

Genotipificación MIRU-VNTR. Se siguió la metodología descrita por Supply, et al. (18), para un análisis de 24 loci. Las PCR se hicieron usando el estuche HotStartTaq DNA Polymerase ${ }^{\mathrm{TM}}$ (Qiagen, Hilden, Alemania). Este método se basa en la amplificación por PCR de un set estándar de 24 loci, con cebadores específicos para cada locus de repetición y en la determinación de los tamaños de los amplicones, los cuales reflejan los números de las copias específicas. Como control positivo y de calidad de las amplificaciones, se usó ADN de la cepa de referencia del complejo $M$. tuberculosis H37Rv (ATCC 27294).

Los amplicones obtenidos se analizaron mediante electroforesis en gel de agarosa (Agarose I ${ }^{\mathrm{TM}}$, Amresco, USA) al $2 \%$ a $110 \mathrm{~V}$ durante una hora. Los tamaños de los amplicones se estimaron por comparación con marcadores de peso molecular de 50 (GeneRuler 50 pb DNA Ladder ${ }^{\mathrm{TM}}$, Thermo Scientific, USA) y 100 pares de bases (pb) (GeneRuler 100 pb Plus DNA Ladder ${ }^{\mathrm{TM}}$, Thermo Scientific, USA). La asignación de los varios alelos VNTR se hizo con el programa GelAnalyzer 2010 (Lazar software, USA).

\section{Interpretación de resultados}

Análisis de relación genética. Las relaciones genéticas entre los aislamientos se visualizaron en el dendrograma obtenido con el algoritmo UPGMA (Unweighted Pair Group Method with Arithmetic Mean) (24) y utilizando el coeficiente de distancia categórica obtenido con las herramientas disponibles en la base de datos MIRU-VNTRplus (https://www.miru-vntrplus. org/MIRU/index.faces).

Análisis de la diversidad alélica de los MIRU-VNTR. La diversidad alélica (h) en un locus dado y la diversidad alélica media $(H)$, se calcularon como $h=$ 1 - $\Sigma x i 2[n /(n-1)]$ y $H=(1 / n) \Sigma h i$, respectivamente, donde xi es la frecuencia de alelo i-ésimo en el locus, hi la diversidad alélica en el locus i y $\mathrm{n}$ el número de loci. También, se calculó el índice de discriminación de Hunter-Gaston. 


\section{Comparación con bases de datos internacionales}

Los resultados obtenidos se compararon con las bases de datos MIRU-VNTRplus (https://www.miru-vntrplus.org/MIRU/index.faces) para la clasificación basada en minisatélites (incluidos los 24 loci MIRU analizados) de los aislamientos dentro de los diferentes linajes conocidos del complejo $M$. tuberculosis, y también con los datos encontrados en la base SITVITWEB (http://www.pasteur-guadeloupe.fr:8081/SITVIT_ONLINE/). Para esta última comparación, se utilizó solamente un set de 12 loci MIRU que ya habían sido descritos por el autor (20).

\section{Aspectos éticos}

El estudio fue aprobado por el Comité Institucional de Revisión de Ética Humana de la Facultad de Salud de la Universidad del Valle y por la Secretaría de Salud Pública Municipal de Santiago de Cali. Los participantes del estudio firmaron un consentimiento informado. Todos los datos y consentimientos firmados fueron conservados en físico y guardados en custodia del investigador principal para mantener la anonimidad de los pacientes. El estudio no interfirió en las actividades rutinarias del programa de tuberculosis relacionadas con el seguimiento de los casos y la administración del tratamiento.

\section{Resultados}

\section{Caracterización sociodemográfica}

La edad promedio de los pacientes era de $40,8( \pm 15,2)$ años, con un rango de 17 a 78 años. La mayoría era de sexo masculino (64,7\%; 68/105) y estaba afiliada al régimen subsidiado de salud (71,2\%; 73/105). En cuanto a sus ocupaciones, las más frecuentes se relacionaban con trabajos no calificados $(20,0 \% ; 21 / 105)$ y con el área de servicio y de ventas $(14,3 \% ; 15 / 105)$. En cuanto a la etnia, el 66,7 \% (70/105) de los pacientes se clasificó en la categoría "otros" y el 14,3 \% (15/105) como población afrodescendiente.

Se incluyeron pacientes de la mayoría de comunas de Cali (con excepción de las comunas 21 y 22), con un paciente por comuna, por lo menos (figura 1). Los resultados detallados con la información sociodemográfica de los pacientes se presentan en el cuadro 1.

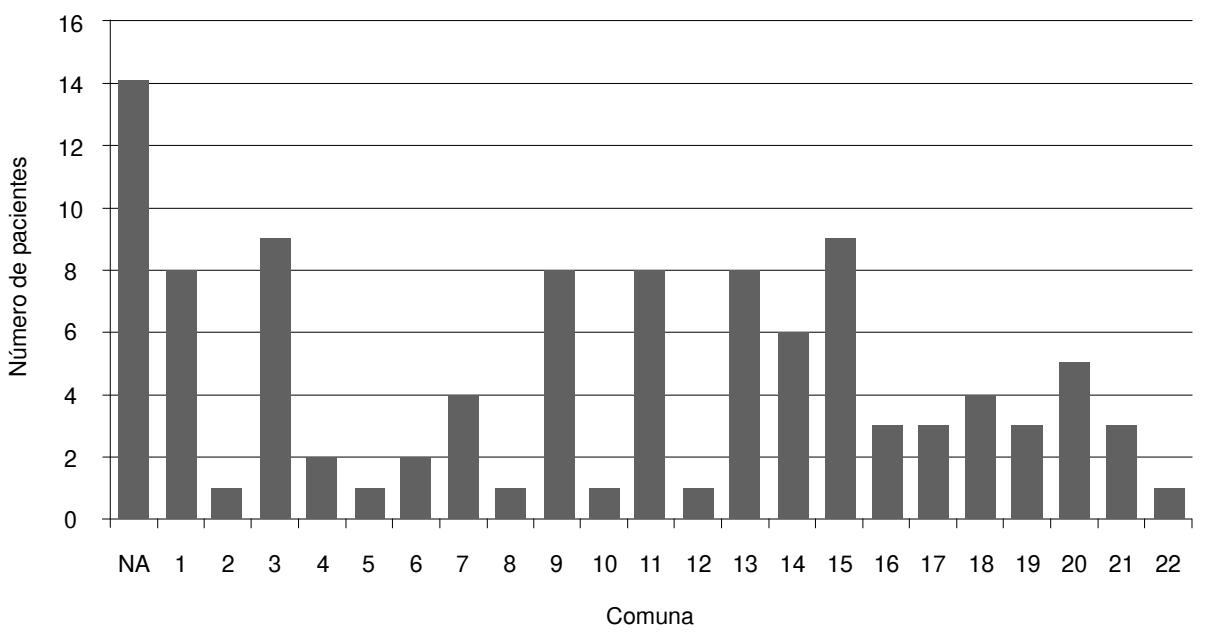

NA: habitantes de la calle, cuya comuna de residencia no estaba registrada.

Figura 1. Distribución por comunas de la población de estudio 
Cuadro 1. Caracterización sociodemográfica de los pacientes, según la Clasificación Internacional Uniforme de Ocupaciones, adaptada para Colombia por el Departamento Administrativo Nacional de Estadística con algunas modificaciones

\begin{tabular}{|c|c|c|}
\hline Variable & $\mathbf{n}$ & $\%$ \\
\hline \multicolumn{3}{|l|}{ Sexo } \\
\hline Masculino & 68 & 64,8 \\
\hline Femenino & 37 & 35,2 \\
\hline \multicolumn{3}{|l|}{ Etnia } \\
\hline Afrodescendientes & 15 & 14,3 \\
\hline Indígena & 1 & 1,0 \\
\hline Mestizo & 3 & 2,9 \\
\hline Otros & 70 & 66,7 \\
\hline Romaní & 1 & 1,0 \\
\hline SD & 15 & 14,3 \\
\hline \multicolumn{3}{|l|}{ Ocupación } \\
\hline Profesionales universitarios & 1 & 1,0 \\
\hline Técnicos & 2 & 1,9 \\
\hline Área de servicios y vendedores & 15 & 14,3 \\
\hline Agricultura, ganadería y pesca & 4 & 3,8 \\
\hline Industria manufacturera, contrucción y minería & 4 & 3,8 \\
\hline $\begin{array}{l}\text { Operadores de instalaciones, de máquinas y } \\
\text { ensambladores }\end{array}$ & 1 & 1,0 \\
\hline Trabajadores no calificados & 21 & 20,0 \\
\hline Desempleados & 26 & 24,8 \\
\hline Estudiantes & 3 & 2,9 \\
\hline En condición de reclusión & 8 & 7,6 \\
\hline SD & 20 & 19,1 \\
\hline \multicolumn{3}{|l|}{ Régimen de salud } \\
\hline Contributivo & 14 & 13,3 \\
\hline Subsidiado & 73 & 69,5 \\
\hline Sin afiliación & 13 & 12,4 \\
\hline SD & 5 & 4,8 \\
\hline
\end{tabular}

$\mathrm{SD} ; \sin$ dato

\section{Caracterización epidemiológica}

El 71,4 \% (75/105) de los casos se habían notificado como nuevos; 10,5 $\%(11 / 105)$ de ellos eran recaídas y 5,7\% (6/105), reingresos después de abandono de tratamiento. El 5,7\% (6/105) de los pacientes fueron seropositivos en la prueba de infección concomitante con HIV y el $18,1 \%$ $(19 / 105)$ se registraron como negativos. Sin embargo, no se registraron resultados de esta prueba en el 76,2 \% (80/105) de los pacientes.

Solo el 8,6 \% (9/105) de los pacientes dijo tener conocimiento de un contacto previo con personas con tuberculosis. El 29,6\% (29/105) de los pacientes presentó alguna condición de riesgo asociada con la tuberculosis, entre ellas, farmacodependencia $(8,6 \% ; 9 / 105)$, diabetes $(4,8 \% ; 5 / 105)$ y desnutrición $(3,8 \% ; 4 / 105)$. En el estudio participaron habitantes de calle $(9,5 \% ; 10 / 105)$ y reclusos $(7,6 \% ; 8 / 105)$. Los resultados detallados de la información epidemiológica se presentan en el cuadro 2.

\section{Sensibilidad a fármacos}

Al analizar los resultados de las pruebas de sensibilidad a los medicamentos, el 85,7\% (90/105) de los casos era sensible a la rifampicina y la isoniacida. El 14,3\% (15/105) presentó resistencia, por lo menos, a uno de estos medicamentos y se notificaron 7 casos $(6,7 \%)$ de tuberculosis multirresistente (ambos medicamentos). 
Cuadro 2. Caracterización epidemiológica de los casos del estudio

\begin{tabular}{|c|c|c|}
\hline Variable & $\mathbf{n}$ & $\%$ \\
\hline \multirow{2}{*}{\multicolumn{3}{|c|}{$\begin{array}{l}\text { Condición de ingreso al Programa de Control de la } \\
\text { Tuberculosis de la Secretaría de Salud Pública de Cali }\end{array}$}} \\
\hline & & \\
\hline Primera vez & 75 & 71,4 \\
\hline Recaída & 11 & 10,5 \\
\hline Reingreso & 6 & 5,7 \\
\hline SD & 13 & 12,4 \\
\hline \multicolumn{3}{|l|}{ Factores de riesgo } \\
\hline Leucemia & 1 & 1,0 \\
\hline Farmacodependencia & 9 & 8,6 \\
\hline Diabetes mellitus & 5 & 4,8 \\
\hline Desnutrición & 4 & 3,8 \\
\hline Desnutrición y farmacodependencia & 6 & 5,7 \\
\hline $\begin{array}{l}\text { Desnutrición, farmacodependencia y enfermedad } \\
\text { pulmonar crónica }\end{array}$ & 3 & 2,9 \\
\hline Embarazo & 1 & 1,0 \\
\hline \multicolumn{3}{|l|}{ Contacto previo } \\
\hline Sí & 9 & 8,6 \\
\hline No & 26 & 24,8 \\
\hline SD & 70 & 66,7 \\
\hline \multicolumn{3}{|l|}{ Prueba de HIV } \\
\hline Positiva & 6 & 5,7 \\
\hline Negativa & 19 & 18,1 \\
\hline SD & 80 & 76,2 \\
\hline \multicolumn{3}{|l|}{ Sensibilidad a medicamentos } \\
\hline Resistencia a la rifampicina & 3 & 2,9 \\
\hline Resistencia a la isoniacida & 5 & 4,8 \\
\hline Multirresistencia & 7 & 6,7 \\
\hline Sensible a los dos medicamentos & 90 & 85,7 \\
\hline
\end{tabular}

\section{Análisis moleculares}

El 60,0\% (62/105) de las muestras de ADN se genotipificaron mediante la técnica MIRU-VNTR, amplificando por PCR los 24 loci estudiados (cuadro 3). En el 35,2\% (37/105) restante, no se obtuvo amplificación ni siquiera en uno de los loci MIRU.

Por otro lado, se observaron alelos múltiples en 5,7\% (6/105) de los aislamientos. El aislamiento 91 presentó alelos dobles en múltiples loci (MIRU 42, 40, 10, 47, 52, 53, ETR-A, ETR-B), por lo cual se sospechó la posible presencia de una infección por más de un genotipo en este paciente. La ficha de notificación para este caso estaba incompleta y los datos registrados no proporcionaron información relevante que pudiera asociarse con esta condición. Para los análisis de diversidad alélica y de relación genética, solo se tuvieron en cuenta los perfiles MIRU de aquellas muestras que tuvieron amplificaciones positivas para todos los 24 loci MIRU y que no presentaron alelos múltiples.

Los loci con la mayor diversidad alélica $(>0,7)$ y mayor capacidad diferenciadora fueron QUB26, QU11b, MIRU 10 y MIRU 40. En estos loci se observó la mayor cantidad de alelos diferentes. En cambio, los loci MIRU 20, MIRU 39, MIRU 46 y MIRU 24 presentaron las menores diversidades alélicas (entre 0,00 y 0,02), y los dos últimos fueron monomorfos, ya que solo presentaron un alelo común en todos los aislamientos (cuadro 4). Con base en estos datos, se obtuvo una diversidad alélica media $(H)$ de 0,461 . 
Cuadro 3. Perfiles MIRU de los aislamientos

\begin{tabular}{|c|c|c|c|}
\hline Aislamiento & Código MIRU & Aislamiento & Código MIRU \\
\hline 1 & 134203332224126153322622 & 63 & 234343322224225153334922 \\
\hline 2 & 234233422324225153333522 & 64 & 242235232424226153131722 \\
\hline 3 & 244212132124116132532322 & 65 & 134204332404136153222622 \\
\hline 4 & 124204332124126153222522 & 67 & 122234332224126153323622 \\
\hline 7 & 134204332214126153322622 & 69 & 234223422424235153233522 \\
\hline 8 & 114204332224116153322622 & 82 & 244214232324116151332232 \\
\hline 9 & 223236332434435153234742 & 92 & 222233332334425153233742 \\
\hline 10 & 214243231634225153339522 & 96 & $13420433241 \mathrm{~s} 4126153222622$ \\
\hline 12 & 134204332224126153322622 & 98 & 234342212444225153334522 \\
\hline 13 & 213245322534435153233732 & 106 & 222234232224226153131722 \\
\hline 14 & 134204232424126153222822 & 111 & 223236332424435153234742 \\
\hline 16 & 134204332444126153522622 & 120 & 134204332224126153322622 \\
\hline 17 & 134204332224126153222622 & 124 & 244214232414156153132232 \\
\hline 18 & 232234232424226153131722 & 142 & 124204332224126153322622 \\
\hline 19 & 223235332534426153334742 & 146 & 244442312344225153334522 \\
\hline 21 & 223234332424435153334742 & 155 & 233236432434435153233742 \\
\hline 22 & 244212132424146142132822 & 159 & 223234322534425153423432 \\
\hline 24 & 213233332534435153233742 & 161 & 134204332224126153322622 \\
\hline 25 & 223233232534425153343742 & 163 & 134204332224126153322622 \\
\hline 26 & 234214232414156153232232 & 168 & 244214232324116153532232 \\
\hline 28 & 232233232424226153331722 & 170 & 334333411443225153323722 \\
\hline 30 & 134203332224126153323622 & 206 & 144205432224126153322632 \\
\hline 33 & 213236332534435153232742 & 213 & 233236332434435153233742 \\
\hline 36 & 224213352944425173342821 & 216 & 242235332424226153131732 \\
\hline 37 & 124204232224126133322622 & 222 & 234132322534225153333222 \\
\hline 39 & 244213231424126183332422 & 224 & 223235332434435153233732 \\
\hline 40 & 234332322344225153134522 & 244 & 134204332225126153322622 \\
\hline 41 & 223235332524425153333742 & 246 & 232224232424226153131722 \\
\hline 42 & 134204332224126153322622 & 249 & 233332312334225153333522 \\
\hline 47 & 233336332534435153233432 & 254 & 252245332424226163131732 \\
\hline 62 & 223235322534435153233732 & 267 & 234213352944425172333823 \\
\hline
\end{tabular}

Cuadro 4. Diversidad alélica por locus MIRU-VNTR

\begin{tabular}{rccccccc}
\hline \multicolumn{1}{l}{ Locus } & $(\mathbf{h})$ & IC & $\mathbf{K}$ & Locus & (h) & IC & K \\
\hline QUB26 & 0,772 & $0,712-0,832$ & 8 & MIRU 31 & 0,506 & $0,431-0.580$ & 3 \\
QUB-11b & 0,758 & $0,702-0,815$ & 7 & VNTR 48 & 0,500 & $0,376-0.624$ & 5 \\
MIRU 10 & 0,742 & $0,676-0,808$ & 5 & MIRU 23 & 0,500 & $0,468-0,532$ & 2 \\
MIRU 40 & 0,710 & $0,648-0,773$ & 5 & MIRU 2 & 0,466 & $0,381-0,551$ & 3 \\
VNTR 52 & 0,698 & $0,633-0,763$ & 5 & 1955 & 0,385 & $0,244-0,526$ & 4 \\
VNTR 42 & 0,677 & $0,600-0,755$ & 5 & MIRU 04 & 0,235 & $0,103-0,368$ & 4 \\
VNTR 47 & 0,662 & $0,624-0,699$ & 3 & MIRU 26 & 0,214 & $0,078-0,349$ & 6 \\
VNTR 49 & 0,653 & $0,575-0,731$ & 5 & MIRU 27 & 0,124 & $0,015-0,234$ & 3 \\
ETRA & 0,627 & $0,531-0,723$ & 6 & MIRU 20 & 0,094 & $0,000-0,190$ & 2 \\
VNTR 43 & 0,565 & $0,471-0,660$ & 3 & VNTR 46 & 0,064 & $0,000-0,148$ & 3 \\
VNTR53 & 0,544 & $0,438-0,651$ & 3 & MIRU 39 & 0,064 & $0,000-0,148$ & 3 \\
MIRU 16 & 0,526 & $0,414-0,638$ & 4 & MIRU 24 & 0,000 & $0,000-0,109$ & 1 \\
\hline
\end{tabular}

(h): diversidad alélica; IC: intervalos de confianza; K: número de alelos diferentes observados; MIRU: Mycobacterial Interspersed Repetitive Units 


\section{Análisis de relación genética}

En la figura 2 se observa el dendrograma obtenido con el algoritmo UPGMA de los aislamientos seleccionados y sus perfiles MIRU. Se obtuvieron 58 perfiles diferentes para los 62 aislamientos. Como puede observarse, solo los aislamientos 12, 42, 120, 161 y 163, provenientes de pacientes diferentes, presentaron el mismo perfil MIRU, lo cual demuestra el gran poder de discriminación de la técnica (HGDI=0,9947).

Conociendo las limitaciones debidas al tamaño de la población, en este punto se decidió no hacer un análisis por conglomerados (agrupaciones formadas por dos o más aislamientos con un mismo patrón) para contrastarlo con las variables sociodemográficas obtenidas, pues los resultados podrían estar determinando relaciones que podían no ser ciertas para la población general de la ciudad.

\section{Comparación con las bases de datos internacionales}

Con base en la comparación de los resultados obtenidos con las bases de datos de MIRU-VNTRplus, los aislamientos estudiados se pudieron clasificar según el linaje filogenético. Primero, se hizo un análisis a partir de la mejor coincidencia (best match) con un punto de corte de distancia por defecto $(0,17)$, el cual presentó una tolerancia de una diferencia de cuatro loci máximo entre los aislamientos comparados. Se encontró que el $58,7 \%$ de los aislamientos fueron asignados a un linaje desconocido, 30,2\% se ubicaron dentro del linaje LAM; 4,8 \% dentro del linaje Haarlem; 3,2 \% fueron asignados al linaje S y, por último, un aislamiento $(1,6 \%)$ se clasificó dentro del linaje Cameroon.

Para clasificar aquellos aislamientos que no fueron asignados a ninguno de los linajes, se hizo un análisis mediante un dendrograma que incluyó las cepas de referencia de la base de datos. Con esta nueva clasificación, finalmente se obtuvo una distribución de 54,8\%(34/62) en el linaje de LAM, de $25,8 \%$ (16/62) en el de Haarlem, de $14,5 \%$ (9/62) en el S, de 3,2 \% (2/62) en el Beijing y de 1,6\% en el Cameroon (1/62) (cuadro 5).

Además, se contrastaron los resultados con la base de datos SITVITWEB, la cual tiene en cuenta el análisis por MIRU-VNTR, pero en su versión de 12 loci. Una vez filtrados los loci adecuados, se encontró que 21 aislamientos $(33,9 \%)$ presentaban patrones huérfanos sin semejanza con ninguno de los perfiles MIRU encontrados en la base de datos (cuadro 5). Los aislamientos quedaron representados por $17 \mathrm{MIT}$ diferentes, los cuales se asignan cuando los perfiles, por lo menos, de dos aislamientos coinciden formando un nuevo grupo. Entre ellos, los más frecuentes fueron el MIT 190 y el MIT 110, con $22,6 \%(14 / 62)$ y $6,5 \%(4 / 52)$, respectivamente. Los demás aislamientos se distribuyeron uniformemente entre los otros MIT encontrados (cuadro 5).

\section{Relación entre las variables sociodemográficas y epidemiológicas y los análisis de relación genética}

Tres de los cuatro casos de pacientes positivos para HIV que se genotipificaron completamente, correspondían al linaje LAM y, solo uno, al linaje Haarlem. No se encontraron correlaciones entre las variables sociodemográficas y epidemiológicas, y la asignación de los aislamientos a un linaje determinado. 
UPGMA-Tree, MIRU-VNTR [24]: Categorical

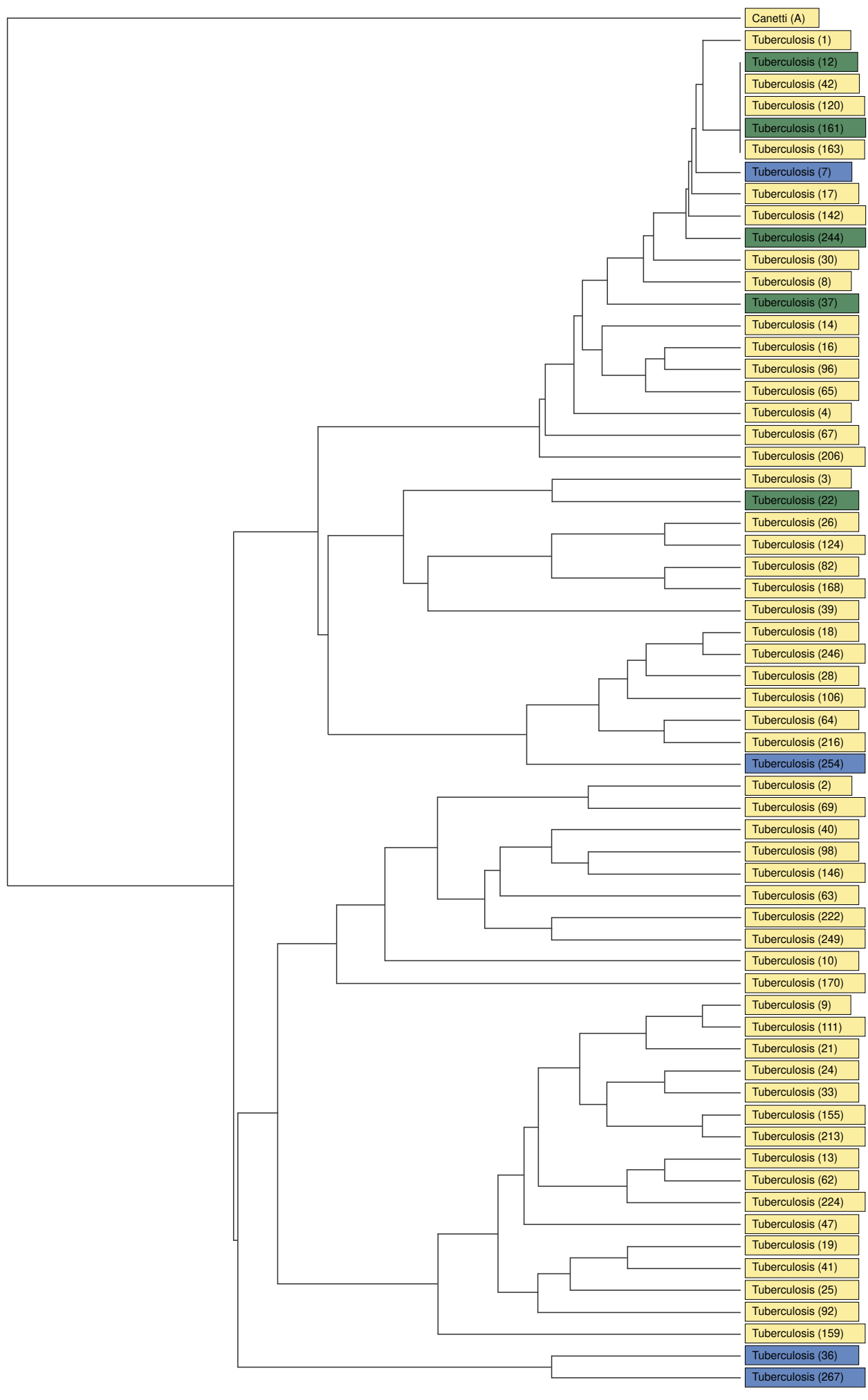

Figura 2. Dendrograma obtenido con el algoritmo UPGMA de los perfiles MIRU. Se resaltan en color verde los aislamientos que presentaron resistencia a rifampicina o isoniacida; en color azul, los aislamientos con multirresistencia. 
Cuadro 5. Distribución por linajes y MIT de los aislamientos del complejo Mycobacterium tuberculosis en la población estudiada

\begin{tabular}{|c|c|c|c|c|c|}
\hline Aislamiento & Linaje & MIT & Aislamiento & Linaje & MIT \\
\hline 1 & $\operatorname{LAM}\left({ }^{*}\right)$ & $\mathrm{h}$ & 63 & $S\left(^{*}\right)$ & 212 \\
\hline 2 & $S(+)$ & 382 & 64 & $\operatorname{LAM}\left(^{*}\right)$ & $\mathrm{h}$ \\
\hline 3 & $\operatorname{LAM}(+)$ & $\mathrm{h}$ & 65 & $\operatorname{LAM}(+)$ & 190 \\
\hline 4 & $\operatorname{LAM}(+)$ & 190 & 67 & $\operatorname{LAM}\left(^{*}\right)$ & $\mathrm{h}$ \\
\hline 7 & $\operatorname{LAM}\left({ }^{\star}\right)$ & 190 & 69 & $S(+)$ & 157 \\
\hline 8 & $\operatorname{LAM}\left({ }^{*}\right)$ & 190 & 82 & $\operatorname{LAM}(+)$ & $\mathrm{h}$ \\
\hline 9 & Haarlem (+) & 110 & 92 & Haarlem $\left({ }^{*}\right)$ & 116 \\
\hline 10 & Cameroon $\left({ }^{*}\right)$ & 263 & 96 & $\operatorname{LAM}(+)$ & 190 \\
\hline 12 & $\operatorname{LAM}\left(^{*}\right)$ & 190 & 98 & $S\left({ }^{*}\right)$ & $\mathrm{h}$ \\
\hline 13 & Haarlem (+) & 46 & 106 & $\operatorname{LAM}(+)$ & 242 \\
\hline 14 & $\operatorname{LAM}(+)$ & $\mathrm{h}$ & 111 & Haarlem (+) & 110 \\
\hline 16 & $\operatorname{LAM}(+)$ & 190 & 120 & $\operatorname{LAM}\left({ }^{*}\right)$ & 190 \\
\hline 17 & $\operatorname{LAM}\left({ }^{*}\right)$ & 190 & 124 & $\operatorname{LAM}(+)$ & 25 \\
\hline 18 & $\operatorname{LAM}\left({ }^{*}\right)$ & 242 & 142 & $\operatorname{LAM}\left(^{*}\right)$ & 190 \\
\hline 19 & Haarlem $\left(^{\star}\right)$ & 247 & 146 & $S(+)$ & $\mathrm{h}$ \\
\hline 21 & Haarlem $(+)$ & 33 & 155 & Haarlem (+) & $\mathrm{h}$ \\
\hline 22 & $\operatorname{LAM}(+)$ & $\mathrm{h}$ & 159 & Haarlem (+) & 187 \\
\hline 24 & Haarlem $(+)$ & 116 & 161 & $\operatorname{LAM}\left({ }^{*}\right)$ & 190 \\
\hline 25 & Haarlem (+) & $\mathrm{h}$ & 163 & $\operatorname{LAM}\left({ }^{*}\right)$ & 190 \\
\hline 26 & $\operatorname{LAM}(+)$ & 25 & 168 & $\operatorname{LAM}(+)$ & 25 \\
\hline 28 & $\operatorname{LAM}\left({ }^{*}\right)$ & 753 & 170 & $S(+)$ & $\mathrm{h}$ \\
\hline 30 & $\operatorname{LAM}\left({ }^{*}\right)$ & $\mathrm{h}$ & 206 & $\operatorname{LAM}(+)$ & $\mathrm{h}$ \\
\hline 33 & Haarlem (+) & 110 & 213 & Haarlem (+) & 110 \\
\hline 36 & Beijing (+) & $\mathrm{h}$ & 216 & $\operatorname{LAM}\left({ }^{*}\right)$ & 247 \\
\hline 37 & $\operatorname{LAM}(+)$ & $\mathrm{h}$ & 222 & $\mathrm{~S}(+)$ & $\mathrm{h}$ \\
\hline 39 & $\operatorname{LAM}(+)$ & $\mathrm{h}$ & 224 & Haarlem $\left({ }^{*}\right)$ & 45 \\
\hline 40 & $S(+)$ & 109 & 244 & $\operatorname{LAM}\left({ }^{*}\right)$ & 190 \\
\hline 41 & Haarlem $\left({ }^{*}\right)$ & 45 & 246 & $\operatorname{LAM}\left({ }^{*}\right)$ & 510 \\
\hline 42 & $\operatorname{LAM}\left({ }^{*}\right)$ & 190 & 249 & $\mathrm{~S}(+)$ & 109 \\
\hline 47 & Haarlem (+) & $\mathrm{h}$ & 254 & $\operatorname{LAM}(+)$ & $\mathrm{h}$ \\
\hline 62 & Haarlem (+) & 45 & 267 & Beijing (+) & $\mathrm{h}$ \\
\hline
\end{tabular}

MIT: MIRU International Types

Los linajes de los aislamientos se designaron según el análisis de mejor coincidencia (best match) $\left({ }^{*}\right.$ ) y el árbol filogenético $(+)$ al compararlos con la información sobre las cepas almacenada en la base de datos MIRUVNTRplus. Se encontraron patrones MIRU huérfanos (h) que no presentaron coincidencias con las cepas notificadas hasta el momento en la base SITVITWEB.

\section{Discusión}

Entre los 62 casos que se genotipificaron completamente mediante la técnica MIRU-VNTR, no hubo una frecuencia alta de transmisión reciente, es decir, grupos de pacientes cuyos aislamientos coincidieran en cuanto a sus perfiles genéticos. Por el contrario, se observó un gran número de genotipos (58 perfiles diferentes) asociados con la circulación de dichos aislamientos en esta población y solo cinco pacientes resultaron agrupados en los análisis (figura 2). Al consultar los datos de estas personas, se encontró que no tenían ningún vínculo epidemiológico y que, además, residían en diferentes comunas de la ciudad. El hecho de no haber encontrado un mayor número 
de casos agrupados, podría explicarse como un efecto del tamaño de la muestra y de la poca representatividad espacial del número de aislamientos recolectados, ya que en muchas de las comunas de donde provenían los casos solo se obtuvieron uno o dos aislamientos.

Sin embargo, este hallazgo resalta la importancia de los estudios estructurados desde el punto de vista epidemiológico, que se complementen con herramientas moleculares como la técnica usada en este y se centren, no solo en la caracterización genética de los aislamientos, sino en la determinación de las posibles cadenas de transmisión, para contribuir al conocimiento de la dinámica de contagio de la tuberculosis pulmonar en la ciudad.

Por ejemplo, en un estudio previo, se evidencia la eficiencia de la técnica MIRU-VNTR para diferenciar los casos de aquellos pacientes que no están relacionados epidemiológicamente, así como su utilidad en estudios poblacionales para una rápida identificación de los aislamientos (25).

En cuanto a la diversidad alélica en cada locus, en este estudio los loci MIRU 10, QU11b y QUB26 fueron los de mayor diversidad alélica y, por ende, mayor poder de discriminación, en tanto que los loci MIRU 20, MIRU 39, MIRU 46 y MIRU 24 fueron los menos diversos. Estos resultados coinciden con los de estudios previos realizados en Bogotá y en diferentes regiones de Colombia, entre ellas, la ciudad de Cali (26-28), lo cual plantearía la posibilidad de escoger un set estándar con un menor número de loci para la tipificación de aislamientos en estudios posteriores que alcancen un poder de discriminación cercano al obtenido por la técnica en su versión de 24 loci.

\section{Distribución de los linajes del complejo M. tuberculosis}

En el análisis de clasificación por linaje de los aislamientos, se encontró un predominio del linaje LAM $(54,8 \%)$, seguido del Haarlem $(25,8 \%)$ y el $S(14,5 \%)$, los cuales son las familias genéticas de mayor prevalencia en la población general del país y de Latinoamérica $(28,29)$. Los resultados obtenidos difieren de los encontrados por Realpe, et al. (27), en cuyo estudio los linajes LAM y Haarlem representaron el 39,1 y el 39,0\%, respectivamente, en los aislamientos provenientes de Cali, en tanto que no se encontró ningún aislamiento del linaje Beijing.

El hallazgo de aislamientos del linaje Beijing (3,2\%) en el presente estudio podría responder a la cercanía de esta ciudad con el puerto de Buenaventura, donde se ha notificado previamente la presencia de este linaje (30).

Resulta atípico, en cambio, el hallazgo de un aislamiento relacionado con el linaje Cameroon, pues hasta el momento no hay estudios que indiquen su presencia en Colombia. Además, este linaje se encuentra asociado con poblaciones africanas (31) y la revisión de los datos del caso no evidenció ninguna correlación importante. Por otra parte, es recomendable hacer un análisis con un segundo marcador y una evaluación más detenida del perfil de resistencia a otros fármacos de este aislamiento para confirmar la validez de esta observación.

En los casos de infección concomitante con el HIV, se encontró con mayor frecuencia el linaje LAM, resultado que coincide con los hallazgos de Castro, et al., en cuyo estudio el $31,7 \%$ de los aislamientos genotipificados pertenecía a esta familia genética (29). Por el contrario, en los estudios de Cerezo, et al., y Beltrán, et al., se encontró un predominio del linaje Haarlem $(26,28)$. Esto indica que se requieren más estudios a nivel nacional para evaluar la dinámica de transmisión de la tuberculosis en esta población. 
En este estudio, se encontraron 21 patrones huérfanos en la comparación con la base de datos del SITVITWEB, es decir, se aportaron nuevos perfiles genéticos basados en la MIRU-VNTR al establecimiento de la estructura poblacional del complejo $M$. tuberculosis en la ciudad. Además, estos patrones huérfanos se compararon con los obtenidos Realpe, et al. (27), (no se presentan los datos) y no se encontraron semejanzas. La genotipificación con otras técnicas, como el spoligotyping o el RFLP-IS6110, puede ser útil para superar ambigüedades en la clasificación y validar los hallazgos, tal como se ha hecho en varias investigaciones en el mundo $(32,33)$.

Asimismo, la comparación con la base de datos del SITVITWEB evidenció la dispersión de los aislamientos entre varios grupos de MIT, y los más frecuentes fueron el MIT 190 y el 110, los cuales se han encontrado también en Bogotá (26). Sin embargo, algunos no se han sido notificado en otras ciudades, lo cual demuestra las posibles diferencias en la distribución de genotipos entre las ciudades de Colombia (cuadro 4).

Sin despreciar el gran aporte que han significado estos estudios, aún quedan muchos vacíos sobre la distribución de los linajes del complejo $M$. tuberculosis y su dinámica de transmisión en Colombia.

\section{Limitaciones del estudio}

A pesar de contar con 62 perfiles MIRU-VNTR dispersos en la zona urbana de Cali, es claro que la muestra estudiada no es representativa de la diversidad genética asociada con la circulación del complejo $M$. tuberculosis en la ciudad, pues se observó una gran variabilidad genética entre los casos estudiados. Sin embargo, los resultados constituyen un aporte al trabajo de vigilancia y seguimiento de la transmisión reciente de aislamientos en la población, así como a la detección de casos de transmisión insospechada en etapas tempranas y de cepas resistentes a los medicamentos.

La mayoría de las fichas de notificación de los casos no estaban completas, lo cual afecta los análisis estadísticos, la interpretación de los datos y las conclusiones que se puedan obtener de este tipo de estudios.

Por otro lado, la alta tasa de casos en los cuales no se obtuvo una amplificación completa de todos los loci MIRU/VNTR, pudo haberse debido a que, en la mayoría de casos, el ADN se extrajo a partir de muestras de esputo. En el estudio de Bidovec-Stojkovič, et al. (34), se evidenció el efecto que tiene la baja densidad de BAAR observados en la baciloscopia en la genotipificación. También, es probable que se encontraran en las muestras inhibidores de la reacción de amplificación.

En conclusión, este estudio permitió evidenciar la distribución de los linajes de aislamientos del complejo $M$. tuberculosis en una población de pacientes residentes en Cali con tuberculosis pulmonar. Se demostró el predominio de los linajes LAM y Haarlem en la población, lo cual coincide con las observaciones de estudios previos. La técnica MIRU-VNTR permitió la identificación de una gran variedad de genotipos que circulaban en la población y, también, de casos de posible transmisión reciente y casos de infección por varios genotipos. En estudios posteriores con una muestra de mayor tamaño y el uso de otros marcadores moleculares, se podrían dilucidar las posibles asociaciones entre la distribución de los linajes y aspectos específicos de la relación patógenohuésped que hayan podido condicionar el predominio estos linajes en la población de la ciudad. 


\section{Agradecimientos}

A Leonor Oviedo, por el apoyo técnico, al Programa de Control de Tuberculosis de la Secretaría de Salud de Santiago de Cali, por la autorización para utilizar las muestras en este estudio, y al Laboratorio de Virología de la Universidad del Valle.

\section{Referencias}

1. World Health Organization. Global tuberculosis control: WHO Report 2017. Geneva: World Health Organization; 2017. p. 224

2. Organización Mundial de la Salud. Preguntas y respuestas: tuberculosis ultrarresistente (TB-XR). Fecha de consulta: 27 de mayo de 2018. Disponible en:

http://www.who.int/features/qa/extensively-resistant-tuberculosis/es/

3. Pedraza L, García C, Muñoz A. Caracterización de pacientes con tuberculosis y tuberculosis resistente a múltiples medicamentos en instituciones de tercer nivel de Bogotá D.C. Enferm Glob. 2012;25:129-38 https://doi.org/10.4321/S1695-61412012000100008

4. Instituto Nacional de Salud. Informe del evento tuberculosis, Colombia, 2017. Fecha de consulta: 27 de mayo de 2018. Disponible en: http://www.ins.gov.co/buscador-eventos/Informesdeevento/TUBERCULOSIS\%202017.pdf

5. Secretaría Departamental del Salud del Valle. Boletín epidemiológico semanal. Semana epidemiológica número 52 de 2015. Fecha de consulta: 10 de mayo de 2015. Disponible en: http://www.valledelcauca.gov.co/salud/descargar.php?id=17080

6. Secretaría Departamental del Salud del Valle. Informe anual, 2016: Vigilancia en salud pública. Fecha de consulta: 27 de mayo de 2017. Disponible en: http://www.valledelcauca.gov.co/salud/descargar.php?id=19934

7. Mathema B, Kurepina N, Bifani P, Kreiswirth B. Molecular epidemiology of tuberculosis: Current insights. Clin Microbiol Rev. 2006;19:658-85. https://doi.org/10.1128/CMR.00061-05

8. Kato-Maeda M, Small P. How molecular epidemiology has changed what we know about tuberculosis. West J Med. 2000;172:256-9.

9. van Soolingen D, De Haas W, Kremer K. Restriction fragment length polymorphism (RFLP) typing of micobacteria. Methods Mol Med. 2001;54:165-203. https://doi.org/10.1385/1-59259-147-7:165

10. Chaoui I, Zozio T, Lahlou O, Sabouni R, Abid M, El Aouad R, et al. Contribution of spoligotyping and MIRU-VNTRs to characterize prevalent Mycobacterium tuberculosis genotypes infecting tuberculosis patients in Morocco. Infect Genet Evol. 2014;21:463-71. https://doi.org/10.1016/j.meegid.2013.05.023

11. Cowan L, Mosher L, Diem L, Massey J, Crawford J. Variable-number tandem repeat typing of Mycobacterium tuberculosis isolates with low copy numbers of IS6110 by using mycobacterial interspersed repetitive units. J. Clin Microbiol. 2002;40:1592-602. https://doi.org/10.1128/JCM.40.5.1592-1602.2002

12. Pitondo-Silva A, Santos A, Jolley K, Leite C, Darini A. Comparison of three molecular typing methods to assess genetic diversity for Mycobacterium tuberculosis. J Microbiol Methods. 2013;93:42-8. https://doi.org/10.1016/j.mimet.2013.01.020

13. Barnes P, Cave M. Molecular epidemiology of tuberculosis. N Engl J Med. 2003;349:114956. https://doi.org/10.1056/NEJMra021964

14. van Embden J, Cave M, Crawford J, Dale J, Eisenach K, Gicquel B, et al. Strain identification of Mycobacterium tuberculosis by DNA fingerprinting: Recommendations for a standardized methodology. J Clin Microbiol. 1993;31:406-9.

15. Rozo J, Ribón W. Molecular tools for Mycobacterium tuberculosis genotyping. Rev Salud Pública (Bogotá). 2010;12:510-21.

16. Cowan L, Diem L, Monson T, Wand P, Temporado D, Oemig T, et al. Evaluation of a two-step approach for large-scale, prospective genotyping of Mycobacterium tuberculosis isolates in the United States. J Clin Microbiol. 2005;43:688-95. https://doi.org/10.1128/JCM.43.2.688-695.2005

17. Kamerbeek J, Schouls L, Kolk A, van Agterveld M, van Soolingen D. Simultaneous detection and strain differentiation of Mycobacterium tuberculosis for diagnosis and epidemiology. J Clin Microbiol. 1997;35:907-14. 
18. Supply P, Allix C, Lesjean S, Cardoso-Oelemann M, Rüsch-Gerdes S, Willery E. Proposal for standardization of optimized mycobacterial interspersed repetitive unit-variable-number tandem repeat typing of Mycobacterium tuberculosis. J Clin Microbiol. 2006;44:4498-510. https://doi.org/10.1128/JCM.01392-06

19. Allix-Beguec C, Harmsen D, Weniger T, Supply P, Niemann S. Evaluation and strategy for use of MIRU-VNTRplus, a multifunctional database for online analysis of genotyping data and phylogenetic identification of Mycobacterium tuberculosis complex isolates. $J$ Clin Microbiol. 2008;46:2692-9. https://doi.org/10.1128/JCM.00540-08

20. Demay $C$, Liens $B$, Burguière T, Hill V, Couvin D, Millet J, et al. SITVITWEB - A publicly available international multimarker database for studying Mycobacterium tuberculosis genetic diversity and molecular epidemiology. Infect Genet Evol. 2012;12:755-66. https://doi.org/10.1016/j.meegid.2012.02.004

21. Shabbeer A, Ozcaglar C, Yener B, Bennett K. Web tools for molecular epidemiology of tuberculosis. Infect Genet Evol. 2012;12:767-81. https://doi.org/ 10.1016/j.meegid.2011.08.019

22. García de Viedma D, Mokrousov I, Rastogi N. Innovations in the molecular epidemiology of tuberculosis. Enferm Infecc Microbiol Clin. 2011;29:8-13. https://doi.org/10.1016/S0213-005X(11)70012-X

23. Organización Panamericana de la Salud. Manual para el diagnóstico bacteriológico de la tuberculosis. Parte 2: Cultivo. Fecha de consulta: 10 de febrero de 2015. Disponible en: http://www1.paho.org/Spanish/AD/DPC/CD/tb-labs-cultivo.pdf

24. Sneath P, Sokal R. Numerical Taxonomy: The principles and practice of numerical classification. San Francisco, CA: Freeman and Company; 1973. p. 549.

25. Rodríguez N, Martínez M, Herranz M, Sánchez M, Barroso P, Bouza E, et al. Evaluation of the new advanced 15-loci MIRU-VNTR genotyping tool in Mycobacterium tuberculosis epidemiology studies. BMC Microbiol. 2008;8:34. https://doi.org/10.1186/1471-2180-8-34

26. Cerezo I, Jimenez Y, Hernández J, Zozio T, Murcia M, Rastogi N. First insight on the population structure of Mycobacterium tuberculosis complex as studied by spoligotyping and MIRU-VNTRs in Bogotá, Colombia. Infect Genet Evol. 2012;12:657-63. https://doi.org/10.1016/j.meegid.2011.07.006

27. Realpe T, Correa N, Rozo J, Ferro B, Gómez V, Zapata E, et al. Population structure among Mycobacterium tuberculosis isolates from pulmonary tuberculosis patients in Colombia. PLoS One. 2014;9:1-12. https://doi.org/10.1371/journal.pone.0093848

28. Beltrán MY. Genotipificación de Mycobacterium tuberculosis en aislados clínicos obtenidos de pacientes VIH positivos de los hospitales Simón Bolívar y Santa Clara de Bogotá (tesis). Bogotá: Universidad Nacional de Colombia; 2016. p. 106.

29. Castro C, Ricardo A, Zabaleta A, Llerena C, Puerto G. Caracterización de aislamientos clínicos de Mycobacterium tuberculosis obtenidos de individuos positivos para HIV en Colombia, 2012. Biomédica. 2017;37:86-95. https://doi.org/10.7705/biomedica.v37i1.3112

30. Nieto L, Ferro B, Villegas S, Mehaffy C, Forero L, Moreira C, et al. Characterization of extensively drug-resistant tuberculosis cases from Valle del Cauca, Colombia. J Clin Microbiol. 2012;50:4185-7. https://doi.org/10.1128/JCM.01946-12

31. Wirth T, Hildebrand F, Allix C, Wölbeling F, Kubica T, Kremer K, et al. Origin, spread and demography of the Mycobacterium tuberculosis complex. PLoS Pathog. 2008;4:1-10. https://doi.org/10.1371/journal.ppat.1000160

32. Barletta F, Otero L, Collante J, Asto B, de Jong B, Seas C, et al. Genetic variability of Mycobacterium tuberculosis complex in patients with no known risk factors for MDR-TB in the North-Eastern of Lima, Perú . BMC Infect Dis. 2013;13:397. https://doi.org/10.1186/1471-2334-13-397

33. Lu W, Lu B, Liu Q, Dong H, Shao Y, Jiang Y, et al. Genotypes of Mycobacterium tuberculosis isolates in rural China: Using MIRU-VNTR and spoligotyping methods. Scand J Infect Dis. 2014;26:98-106. https://doi.org/10.3109/00365548.2013.858182

34. Bidovec-Stojkovič U, Seme K, Žolnir-Dovč M, Supply P. Prospective genotyping of Mycobacterium tuberculosis from fresh clinical samples. PLoS One. 2014;9:e109547. https://doi.org/10.1371/journal.pone.0109547 